\title{
El constructivismo geográfico y las aproximaciones cualitativas ${ }^{1}$
}

\author{
Alicia Lindón ${ }^{2}$
}

\begin{abstract}
RESUMEN
Este trabajo presenta una aproximación cualitativa denominada hologramas socioespaciales, destinada al estudio de la construcción social de los lugares. Esta propuesta es parte de una investigación de mayor alcance centrada en la espacialidad de la vida cotidiana en contextos urbanos, más específicamente periféricos y suburbanos. En esta orientación, la espacialidad es el núcleo central de la investigación, a través del concepto de lugar tal como es entendido en la perspectiva de la geografía humanista. Esto implica que la investigación se asume como una perspectiva comprensiva de la vida cotidiana, aunque con un sesgo constructivista: se concibe al lugar como una construcción social siempre en curso, necesariamente inacabada.

En la primera parte de este trabajo se presenta un acercamiento muy somero al lugar como un tipo de mirada particular para estudiar la espacialidad de la vida social. Luego, en el segundo apartado, se profundiza la cuestión del lugar a partir de la propuesta de estudiarlo como una construcción social. Posteriormente se desarrollan los elementos básicos de la aproximación cualitativa denominada hologramas socioterritoriales. En el apartado siguiente se presenta un holograma particular, en el contexto de la periferia excluida del oriente de la ciudad de México. Por último, terminamos con unas reflexiones abiertas.
\end{abstract}

Palabras clave: Hologramas socioespaciales, geografía humanista, lugares invisibles.

\begin{abstract}
This paper presents a qualitative approach named "socio-spatial holograms", focused on analyzing the social construction of places. This proposal is part of a broader research centered on the spatiality of everyday life in urban contexts; more specifically the peripheral and suburban's environments. In this line, spatiality is the core of this research, through the concept of place, as understood by the humanistic geography. This implies that the research is oriented in a comprehensive perspective of everyday life, but with a constructivist bias: place is conceived as a social construction always in transformation or on the move, never finished.

In the first part of this work, a basic approach to the concept of place is presented, as a particular gaze to study the spatiality of social life. Then, in the second part, the place question is deepened, proposing the possibility to study it as a social construct. After that, the basic elements of this qualitative approach named "socio-territorial holograms" are lay down. In the next part, a particular hologram is presented, in the context of the marginal periphery of eastern Mexico City. Finally the paper concluded with some open remarks.
\end{abstract}

Key words: Socio-spatial holograms, humanistic geography, invisibles places.

\footnotetext{
1 Artículo recibido el 10 de marzo de 2007 y aceptado el 1 de mayo de 2007
}

\footnotetext{
2 Universidad Autónoma Metropolitana, Campus Iztapalapa, Ciudad de México (México). E-mail: alicia.lindon@gmail.com
} 
Este trabajo plantea el tema del constructivismo geográfico como una forma de aproximarse al estudio del espacio, que puede ponerse en práctica a través de metodologías cualitativas. En este sentido, se desarroIla una propuesta metodológica cualitativa particular que hemos denominado hologramas socioespaciales, destinada al estudio de la construcción social de los "lugares". Esta propuesta se articula dentro de nuestro trabajo de investigación que de manera general, se aboca a estudiar la espacialidad de la vida cotidiana en contextos urbanos, más específicamente periféricos y suburbanos. En esta línea de trabajo hemos enfocado la espacialidad a través del concepto de "lugar" tal como es entendido en la perspectiva de la geografía humanista. Entre otras cosas, esto implica que nuestro trabajo se asume enteramente como una perspectiva comprensiva de la vida cotidiana, aunque con un sesgo constructivista: concebimos al lugar como una construcción social siempre en curso, necesariamente inacabada.

De esta forma, en la primera parte de este trabajo se presenta un acercamiento muy somero al lugar como un tipo de mirada particular para estudiar la espacialidad de la vida social. Luego, en el segundo apartado, profundizamos la cuestión del lugar a partir de la posibilidad de estudiarlo como una construcción social. Posteriormente desarrollamos los elementos básicos de nuestra aproximación cualitativa que denominamos "hologramas socioterritoriales". En nuestro propio trabajo de investigación los hemos utilizado en diversas ocasiones, por ejemplo para abordar la construcción social de espacios del miedo, pero también para estudiar la construcción social de paisajes parcialmente invisibles (Lindón, 2007). En el apartado siguiente presentamos un holograma particular, contextualizado en la periferia excluida del oriente de la ciudad de México. Por último, terminamos con unas reflexiones abiertas.

\section{El lugar como orientación general}

El lugar refiere a una localización concreta, por eso los geógrafos teoréticos (cuantitativos) lo consideraban un punto, y entre varios puntos es posible medir distancias. Sin embargo, las geografías humanistas anglosajonas - desde los años setenta- reconstruyeron este concepto planteando que los lugares no solo son una localización particular, también tienen identidad, o bien, tienen rasgos peculiares a través de los cuales son identificados, se les atribuyen valores y son objeto de construcción simbólica. La revisión de esta discusión teórica indudablemente es una labor relevante, atractiva y también ardua, pero no es nuestro objetivo en esta ocasión. Antes bien remitimos a algunas obras de la extensa literatura sobre el tema. Baste en esta ocasión solo con especificar brevemente a qué nos referimos con las expresiones próximas entre sí de lugar y espacio vivido.

El concepto de lugar hace referencia a espacios delimitados, con límites precisos, que para los sujetos representan certezas y seguridades otorgadas por lo conocido (Tuan, 1977). A pesar de que el lugar alude a un espacio con límites, dichos límites se extienden hasta donde lo hace el contenido simbólico de los elementos objetivados en él y que pueden ampliarse a través de redes y relaciones de sentido. Por ello, se puede considerar al lugar, siguiendo a Gumuchian (1991), como "una acumulación de significados", o bien, en las palabras similares de Entrikin (1976), el "lugar es un depositario de significados". La acumulación de significados sobre un lugar también ha sido estudiada a través de "íconos portadores de sentido que se lo confieren a los lugares", como lo señalara el geógrafo francés Joel Bonnemaison (2000).

En las geografías francófonas, también desde los años setenta - de manera casi simultánea a lo ocurrido en las geografías anglosajonas-, se viene desarrollando el concepto de espacio vivido, con un contenido más o menos próximo al que ha tomado el de lugar y sentido del lugar en la geografía anglosajona. El concepto de espacio vivido (espace vécu) fue propuesto en 1976 por Armand Frémont (1999). Así para la geografía de Francia del Oeste $^{3}$ :

\footnotetext{
3 Este núcleo de la Francia del Oeste (Caen, Angers, Le Mans, Nantes, Rennes) reunió a Armand Frémont, Jacques Chevalier, Robert Hérin y Jean Renard (Marconis, 1996, p. 189).
} 
"La propuesta del espacio vivido no se limita a reconocer lugares frecuentados, definir itinerarios, situar al hombre-habitante en su cuadro familiar de existencia (...) sino focalizar la mirada en la relación con las representaciones (...), es decir superar el espacio extensión (o espacio-soporte), para abordar la noción de representación (imagen) del espacio, planteando una nueva pregunta: ¿cómo ven los hombres el espacio? (...) El espacio vivido es reivindicado como un espacio cargado de valores (Chevalier, 1974: 1)"

Años más tarde, Guy Di Meo -otro geógrafo francés- también toma parte en esa reflexión inicial. Di Meo diferencia el espacio de vida del espacio vivido, y completa la propuesta teórica con el concepto de espacio social:

"El espacio de vida se confunde, para cada individuo, con el área de sus prácticas espaciales. Es el espacio frecuentado por cada uno de nosotros, con sus lugares atractivos, sus nodos en torno a los cuales se construye la existencia individual: la morada, la casa, los lugares de trabajo y de ocio. El espacio concreto de lo cotidiano (1991: 123)".

Por otra parte, Di Meo -siguiendo la tradición de Frémont- ha planteado que:

"El edificio construido sobre las bases de la materialidad y sus prácticas (el espacio de vida) se enriquece de la pulpa de los intercambios sociales (el espacio social), de las cargas emotivas, de las imágenes y de los conceptos individuales, aunque siempre son de esencia social, que forjan nuestra representación del mundo sensible y contribuyen a conferirle sentido (espacio vivido) (1991: 127)"

Esta forma más compleja e integradora es el espacio vivido. Por ello para Frémont (2005: 79), "el espacio vivido es el más completo, el más denso, el que integra todas las distancias y todas las complejidades".

A partir de estas dos líneas teóricas, sobre el lugar (desde el pensamiento anglo- sajón) y el espacio vivido (desde el pensamiento francófono), ha emergido un abanico muy amplio de problemáticas espaciales de investigación en ambas tradiciones del pensamiento. Por ejemplo, algunas buscan "reconocer lugares frecuentados, definir itinerarios, situar al hombre-habitante en su cuadro familiar de existencia" (Gumuchian, 1991: 62). Asimismo, el concepto de lugar ha abierto un importante horizonte en torno al estudio de la identidad del lugar (Hiernaux, 2000; Nogué, 1999) y el sentido del lugar (sense of place) (Butz \& Eyles, 1997). Otra derivación del concepto del lugar, son los estudios sobre la memoria del lugar y los lugares de memoria, para retomar la expresión que ha hecho célebre Pierre Nora (Nora, 1997; Javeau, 2000). Otros ángulos abiertos a partir del concepto de lugar son las denominadas fantasías geográficas (Rowles, 1978), las topofilias, topofobias y los paisajes del miedo (Tuan, 1990; 1980; Lindón, 2005b; Yori, 1998; 1999; Uribe Castro, 2002), los lugares y paisajes efímeros y fugaces (Hiernaux, 2006), asimismo la tan polémica deslugaridad o placelessness planteada inicialmente por Relph (1976), la "atopía" (Turco, 2000; Lindón, 2005a), la territorialidad, la apropiación de los lugares y los lugares mágicos y míticos (Sack, 1980; 1986; Raffestin, 1977; 1982; 1986), los espacios domésticos e interiores (Collignon \& Staszak, 2003; Lindón, 2006b); así como el papel de la retórica y sus tropos en la construcción de los lugares (Debarbieux, 1995; Lindón, 2003). También esta visión del lugar ha producido una profunda renovación de los estudios del lugar y el paisaje con énfasis cultural (Nogué, 1989; 1992; 2006a; 2006b; Cosgrove, 1985; 2002; Duncan \& Ley, 1993), muchas veces articulados a la indagación sobre identidades. En esta última perspectiva, lo innovador radica en no concebir al paisaje como simple producto cultural (como se hizo dentro de la tradición saueriana), sino reconocer la anterior condición de producto cultural junto a su capacidad para incidir en la cultura.

La consolidación de estas miradas egocéntricas -desde el sujeto y su subjetividad (Hiernaux \& Lindón, 2004)- las geografías de los mundos interiores (Debarbieux, 1997), así como el microanálisis, parecería que desdibuja las fronteras rígidas entre el 
pensamiento francófono y anglosajón, al menos en este campo de trabajo. Así, nuestro trabajo aspira a ubicarse en esa convergencia de las dos tradiciones del conocimiento: sobre el lugar y el espacio vivido.

\section{La construcción social del lugar/espacio vivido}

Esta concepción del lugar nos ha llevado a esa corriente de la teoría social actual conocida como constructivismo. Si bien es una perspectiva ampliamente reconocida en la filosofía de las últimas décadas ${ }^{4}$ y en las diversas ciencias sociales y las humanidades, es menor el impacto que ha tenido en el caso particular de la geografía y en general en el estudio de la espacialidad. Efectivamente, los autores que se asocian usualmente al constructivismo -tales como Bateson, Gergen, Watzlawick, Maturana, Varela, Berger, Luckmann, Schutz- en principio no parecen tener vínculos con la geografía y los estudios territoriales. Sin embargo, desde hace tres décadas el humanismo geográfico viene abriendo un camino que actualmente parece fértil para el diálogo con estas líneas del pensamiento, sobre todo por el replanteamiento del concepto de espacio (space) en el de lugar (place) y en el de espacio vivido (espace vécu), y también por otorgarle centralidad a la experiencia espacial (Buttimer \& Seamon, 1980). Algunos autores, han insistido más en la dimensión individual y la intencionalidad del individuo (Buttimer, 1976), con un énfasis de tipo fenomenológico. Otros -con una mirada más próxima al interaccionismo simbólico, sin perder la componente fenomenológica- han colocado el énfasis en la negociación cotidiana de las personas en contextos concretos (Ley, 1978). En esta última perspectiva, cabe destacar que David Ley (1978) plantea, temprana-

\footnotetext{
4 Aunque la expresión constructivismo es reciente -muchos marcan el hito inicial en 1980- remite a una problemática del conocimiento muy antigua, como es la relación entre el objeto y el sujeto que conoce. Hay antecedentes en el mundo griego clásico, en el siglo XVII con Gianbattista Vico, en el siglo XVIII con Inmanuel Kant, a fines del XIX con William James y Friedrich Nietzsche, en la primera mitad del XX con José Ortega y Gasset y Alfred Schutz, por nombrar solo algunas figuras prominentes.
}

mente, la perspectiva de la construcción social de la realidad desde la espacialidad y posteriormente incorpora directamente la construcción social del lugar desde varias entradas: por ejemplo, el intercambio simbólico y recíproco entre la gente y los lugares (Ley, 1981a); o bien, la convergencia de la subjetividad y la intersubjetividad con la materialidad de los lugares, o la articulación de lo subjetivo y lo objetivo (Ley, 1989).

Indudablemente, el geógrafo sueco Gunnar Olsson también ha jugado un papel central en esta perspectiva de constructivismo espacial por su trabajo sobre la inclusión del lenguaje, en particular los juegos del lenguaje de Wittgenstein en el estudio de la espacialidad (Olsson, 1978; 1980; 1991). Según Olsson (1997: 39), "Los límites del ecúmene son los límites de mi mundo. Los límites de mi mundo son los límites de mi lenguaje. Los límites de mi lenguaje son pensamiento-y-acción al límite de sí mismo". Asimismo, la concepción de los lugares como textos, la textualidad (Barnes \& Gregory, 1997: 138-144) Ilevó a incluir planteamientos como el de la intertextualidad, inspirados en la obra de Todorov y Bakhtin: un lugar evoca otro lugar, entre un lugar y otro se da una relación de copresencia, el sentido de un lugar evoca al sentido de otro lugar. Asimismo, estas miradas constructivistas también introdujeron en la geografía el problema de las representaciones (Bailly, 1989) y el consecuente tema de la crisis de las representaciones (Duncan \& Ley, 1993: 4). La crisis deriva tanto del sentido literal de representar como reproducción fiel de lo real (las representaciones miméticas), como también del tipo de representaciones legitimadas, sobre todo a través de la racionalidad cartográfica (Harley, 2005; Minca, 2002).

Todos estos aspectos han sido retomados y problematizados por el movimiento denominado cultural turn, que desde fines de los años ochenta mueve a la geografía anglosajona (Philo, 1999). Dicho Giro Cultural ha tomado como uno de sus principales ejes la incorporación de lo no material en el estudio del espacio y la espacialidad. En estas miradas cabe destacar aportes como el de Derek Gregory que buscan la articulación de la mirada de Lacan en relación con la es- 
pacialidad (Gregory, 1995). No obstante, tanto Derek Gregory como Nigel Thrift y Don Mitchell (voces destacadas a favor de la semiótica del espacio a fines de los ochenta), después de haber impulsado la consideración del lenguaje, lo textual y lo semiótico, a mediados de los noventa proponen abandonar este camino para centrarse en las "prácticas" que construyen el lugar.

Desde la geografía francófona, aunque muy imbuida del pensamiento anglosajón, las palabras de Antoine Bailly también resultan elocuentes de estas miradas que rechazan la reducción del espacio a su componente material:

"En un entorno histórica y socialmente dado, el individuo construye su propia realidad articulando lo estructural, lo funcional y lo simbólico; (...) el paisaje nos remite a nuestra experiencia existencial; cómo se articulan lo real y lo imaginario en cada lugar (1989: 11)".

Estas perspectivas muestran que el problema de la espacialidad de la vida social no debería reducirse a una realidad material, única y externa a las personas. Más bien, siguiendo a Antoine Bailly (1989), se lo puede entender en la mezcla entre lo imaginario y lo real.

En suma, el estudio de la espacialidad también puede asumir el constructivismo ya que uno de sus presupuestos es que lo que conocemos y creemos resulta del lenguaje con el que entendemos y transmitimos nuestras percepciones del mundo. Los constructivistas han dicho que al hablar -en un mundo compartido siempre con otros- creamos la realidad. Por eso, un mismo fenómeno, una misma realidad, puede ser construida de diferentes formas en función de distintos puntos de vista.

Si bien en ciencias sociales como pueden ser la sociología o la psicología social, el tema del sujeto y su punto de vista ya cuenta con tradiciones consolidadas, en el estudio de la espacialidad y del territorio la situación es diferente: existen antecedentes relevantes (voces pioneras), pero distan de ser enfoques instituidos y retomados en las comunidades académicas. Posiblemente, una de las circunstancias que ayude a comprender estos rezagos y resistencias sea el peso que ha tenido la idea del espacio como una realidad material objetiva. Aunque los aportes de muchos autores han mostrado que el espacio y el territorio también son objeto de elaboración subjetiva por parte del sujeto-habitante, las resistencias a su inclusión -más aún, de manera central- son considerables.

En nuestra propuesta, el constructivismo geográfico -o espacial- se puede definir al menos desde los ejes que planteamos a continuación, considerando que no son excluyentes entre sí, sino distintas entradas que se entrecruzan:

- El espacio visto a la luz del sujeto como habitante (Dardel, 1990).

- El lugar en la dialéctica entre el espacio y la sociedad. Esto significa que el lugar es concebido como productor de lo social y al mismo tiempo como producto social. Así, el lugar resulta de un movimiento constante entre fuerzas constituyentes y constituidas: los sujetos construyen el lugar y el lugar es construido socialmente, el lugar modela la vida social que allí se aloja.

- El lugar en la dialéctica entre las formas espaciales y los sentidos del lugar. Esto implica que el lugar se despliega en lo material y en lo no material. Así, lo material resulta de las formas de sentir, percibir y concebir los elementos materiales del entorno de algunos sujetos sociales.

- Las redes que articulan lugares -incluso distantes- a partir de la experiencia de los habitantes de esos lugares.

En esta concepción, el lugar/espacio vivido construido socialmente queda totalmente vinculado a la lógica del movimiento como una tensión constante entre las permanencias y los cambios, que a su vez pueden operar en distintas escalas temporales y espaciales. En otros términos, la construcción del lugar puede llevar consigo permanencias breves o extensas en su duración, así como permanencias de gran extensión espacial o reducidas espacial- 
mente. Lo mismo puede ocurrir con los cambios, pueden ser rápidos, es decir ocurrir en tiempos muy breves o desarrollarse en temporalidades largas. De igual forma, el lugar puede construirse a través de cambios registrados en extensiones espaciales reducidas o en extensiones extensas. Asimismo, los cambios o permanencias pueden ser en las formas materiales como en los sentidos del lugar.

De esta forma no es posible considerar al lugar como una realidad estable, inmanente e inmutable. En algunos casos se Ilega a constatar la permanencia. Esto sobre todo se asocia con tres circunstancias: desde el punto de vista del sujeto, con la repetición del hacer; desde la dialéctica espacio-sociedad (Santos, 1990), con la capacidad del espacio para modelar a las sociedades; $y$, desde la dialéctica formassentidos (Eyles, 1985; Ley, 1981a; 1981b), con el peso de las formas para definir los sentidos del lugar.

En el otro extremo, la lógica del movimiento también supone la posibilidad de cambio constante en el lugar. Desde la perspectiva del sujeto-habitante, el cambio en el lugar resulta de cambios en sus formas de hacer, en sus prácticas. Desde la dialéctica espacio-sociedad, el cambio resulta de la posibilidad de que la sociedad -a través del sujeto- pueda recrear, transformar, modificar, el lugar en todo momento. Desde la dialéctica formas-sentidos, el cambio emerge de la posibilidad de que el sujeto le asigne nuevos sentidos a unas mismas formas espaciales.

Entre estas dos lógicas opuestas de cambio y permanencia en distintas escalas de tiempo y espacio, la construcción social del lugar se objetiva en numerosas situaciones intermedias entre el cambio y la permanencia, entre lo reducido y lo extenso espacialmente, entre lo efímero y lo duradero en el tiempo. En estas lógicas de cambio y permanencia a través de las cuales se da la construcción social de los lugares, su expresión más evidente es el lenguaje. Sobre todo en lo relacionado con los sentidos atribuidos a las formas espaciales, aunque también interviene en la definición de las formas materiales mismas.

\section{Las aproximaciones cualitativas y los hologramas socioespaciales}

Las miradas cualitativas atentas a lo minúsculo, aun cuando sea fugaz, permiten un acercamiento fértil a las prácticas espaciales y su intencionalidad, así como a los sentidos otorgados a los lugares (Pred, 1981; 1984; Ley, 1981a; Daniels, 1997). Por su parte, las prácticas son parte de las formas espaciales, no como formas fijas sino en tanto configuraciones en movimiento ${ }^{5}$. Por ello, las aproximaciones cualitativas y el microanálisis, al conectar lo material y lo simbólico (Eyles, 1988), constituyen un camino idóneo para estudiar empíricamente la construcción social del lugar a partir de las prácticas espaciales (Ley, 1988; Eyles, 1985).

Nuestra propuesta consiste en demarcar, dentro de los discursos de los habitantes del lugar, hologramas socioterritoriales que nos permitan comprender la construcción social de lugares/espacios vividos particulares. La idea del holograma procede de la física. Nosotros la estamos tomando de manera metafórica. De acuerdo a la invención realizada en 1947 por el físico húngaro Dennis Gabor, la holografía es una técnica avanzada de la fotografía por la cual "en la placa fotográfica se impresionan las interferencias causadas por la luz reflejada de un objeto con la luz indirecta. Iluminada (después de revelada) la placa fotográfica con la luz del láser, se forma la imagen tridimensional del objeto original" (RAE, 1992: 1117).

Es metafórico el uso que hacemos de la expresión hologramas, ya que en esencia no nos estamos refiriendo ni a placas fotográficas, ni a luces ni a rayos láser. No obstante, consideramos que la metáfora puede resultar iluminadora del sentido que se busca poner de manifiesto: A través de un procedimiento reconstruir una imagen que de otra forma no se percibe. En la holografía se trata de un procedimiento técnico de iluminación. En esta versión del constructivismo

\footnotetext{
5 Cabe recordar que Soja (1996) denomina prime espacio a la conjunción de las formas espaciales materialmente dadas y las prácticas espaciales.
} 
geográfico se trata de un procedimiento de interpretación. La holografía obtiene con ese procedimiento una imagen tridimensional, es decir, de mayor profundidad y en consecuencia, más próxima al objeto mismo. En nuestro constructivismo geográfico, la interpretación nos permite obtener una imagen más profunda de los lugares: es decir no solo la descripción de sus rasgos materiales y evidentes, sino también los sentidos que destacan unos rasgos del lugar y desdibujan otros.

Nuestros hologramas cualitativos funcionan de la siguiente manera: los habitantes del lugar producen las narrativas de vida en situación de interacción con el investigador. Esto constituye el material básico y objeto de análisis, es decir el equivalente de la placa fotográfica tradicional, en las cuales la luz deja una huella. Por su parte, a la acción de iluminar con un rayo láser que se aplica a los hologramas físicos, equivale en nuestros hologramas socioespaciales la tarea de descifrar y decodificar lo que está detrás de las palabras, es decir los significados más profundos. Las conexiones entre un significado y otro nos permiten reconstruir una trama de significados que está encriptada en el discurso. Esa trama permite conectar distintos lugares no visibles para una observación superficial. Esta última tarea de conectar en una trama de significados, metafóricamente corresponde al momento en el que el físico logra la imagen tridimensional por la iluminación del rayo láser.

Cabe señalar que el desciframiento de esta "tercera dimensión" exige que el observador asuma cierta posición, de la misma forma que la tercera dimensión del holograma físico exige mover el mismo, o tomar cierto ángulo que permita visualizar la tercera dimensión. En otros términos, no es posible desvelar esta dimensión, en principio oculta, desde cualquier posición. En este sentido, la práctica de investigación muestra que un análisis tradicional de los discursos no permite descifrar todo lo que "esconden detrás" (incluidos los lugares invisibles), sino que es a través de prácticas analíticas cualitativas profundas que podremos encontrarlas.

Todo lo anterior se funda en el supuesto (nuestra posición epistemológica) que el lenguaje materializa el flujo de la vida social, le da una forma y lo cristaliza en ella, aun cuando lo cotidiano sea un discurrir que nunca se detiene ni adopta formas estables, sino formas cambiantes. Las formas lingüísticas, las palabras empleadas, le dan formas particulares a lo multiforme que es lo vivido (Pratt, 1997; Mondada, 2000; Nogué \& Villanova, 1999). Así como la placa fotográfica "inmoviliza" la vida social que fluye continuamente. La narrativa hace lo propio a través de las formas lingüísticas empleadas: las palabras empleadas "inmovilizan" lo vivido. Entonces, al trabajar con fragmentos del lenguaje (las narrativas), esto nos permite captar momentos de la vida social, capturar el instante dentro del flujo, en el sentido de Bergson. En términos de un constructivismo geográfico, esto nos permite captar esos lugares no visibles para quien no participa de cierta experiencia, o bien los paisajes fugaces de los que habla Hiernaux (2006), que en última instancia son instantes del flujo de la vida social.

Los hologramas socioespaciales se dibujan dentro de narrativas de circunstancias en apariencia banales, pero de gran valor metodológico por condensar elementos claves acerca de la construcción del sentido del lugar. En consecuencia, proporcionan pautas acerca de la forma en que la persona se relaciona con ese lugar y con otros lugares.

En los hologramas socioespaciales, el narrador refiere a lugares y prácticas concretas que allí se despliegan, verdaderos escenarios espacio-temporales de la vida cotidiana (Lindón, 2006a). Por medio de las prácticas la persona se apropia del lugar para distintos fines, compartiéndolo e interactuando con otros que convergen en el lugar. Sin embargo, el holograma socioespacial no es un simple relato de prácticas y lugares, o un relato de simples escenarios en los que se despliegan prácticas. Es un relato de prácticas, lugares y escenarios que contiene dentro de sí y de manera encapsulada, otros lugares, sentidos de los lugares, intencionalidades de los habitantes de esos lugares, simbolizaciones de los lugares y del quehacer que en ellos se concreta.

En esta perspectiva, el holograma socioespacial es una propuesta metodológica 
abierta a la interpretación espacial (Ley, 1988) en dos planos: una localizada y otra desplegada en una red de lugares interconectados a través de lo vivido (Di Meo, 1999). A continuación se analizan ambas posibilidades de la interpretación espacial.

El primero de estos planos de la interpretación espacial -la localizada- toma como punto de partida el lugar en sí mismo en el cual se ancla la narrativa, y desde allí llega a otro lugar invisible aparentemente, que está superpuesto con la forma espacial del lugar directamente referido. El lugar invisible está construido simbólicamente sobre las formas espaciales enteramente visibles. Esa construcción simbólica conlleva una apropiación particular a través de la realización de prácticas no esperables de acuerdo a las formas espaciales visibles, pero sí esperadas con relación a su construcción simbólica. Un ejemplo de esto son ciertas prácticas que suelen realizar los homeless en la vía pública, como puede ser la de dormir. Esta práctica puede no ser esperable si se observa el lugar superficialmente como parte de una acera o del espacio público. Sin embargo, es esperable si se comprende que ese habitante Ilamado homeless ha apropiado ese lugar como su hogar (Zeneide-Henry, 2002).

En este primer nivel el holograma socioespacial permite una interpretación espacial que va más allá del descifrar el sentido del lugar: permite reconstruir dos lugares físicamente superpuestos, pero uno de ellos de visibilidad evidente mientras que el otro es de visibilidad parcial, o lo que es lo mismo, casi invisible.

El segundo plano de la interpretación espacial que permite el holograma socioespacial es el que establece conexiones entre el lugar en el cual está anclada la narrativa (ya sea el lugar evidente o el parcialmente visible) y otros lugares distintos (incluso, pueden ser diametralmente diferentes) y distantes. Estas redes o conexiones entre lugares se establecen a través de las experiencias de vida del sujeto: son lugares que integran el acervo de experiencias espaciales de un habitante (Di Meo, 2000b; 1999).

La condensación que ha realizado el lenguaje en estos hologramas socioespaciales permite encontrar huellas susceptibles de interpretación en dos perspectivas: una, respecto a la particular superposición de lugares. La otra, en cuanto a situaciones ancladas en lugares distantes de aquellos a los cuales se refieren los hologramas directamente, pero que en algún aspecto están conectadas con ella. Esto puede entenderse como una expresión de la intertextualidad espacial: un lugar evoca otro lugar. Por ello, los hologramas socioespaciales permiten comprender -al menos parcialmente- algunas formas de relación del sujeto con su espacio de vida que van más allá de una situación particular u otra. En otras palabras, los hologramas socioespaciales tienen la virtud de dar cuenta del cruce de lo situacional y particular con lo que se reitera en distintas situaciones y a través del tiempo. Esto constituye una forma de la singularidad o betweenness (Entrikin, 1991).

En suma, este recurso metodológico tiene una enorme potencialidad porque permite observar lo minúsculo para, desde allí, desentrañar las tramas de significación y los lugares parcialmente visibles en los que estas se objetivan. No obstante, también hay que tener en cuenta que es un recurso metodológico inscripto en la lógica del rompecabezas necesariamente incompleto, la lógica de los fragmentos densos pero siempre parciales. En última instancia, el análisis de los significados en contextos específicos (Eyles, 1988).

A continuación presentamos un holograma particular que corresponde a narrativas de jóvenes habitantes de la periferia excluida del oriente de la ciudad de México. En esta zona son frecuentes las formas espaciales típicas de la pobreza urbana, reiterada en las periferias excluidas de las ciudades latinoamericanas. Al nivel de las formas espaciales se trata de espacios muy conocidos y ampliamente estudiados. Posiblemente en ese nivel de las formas espaciales, los hologramas no agregan demasiado respecto a lo conocido. Sin embargo, desde la perspectiva de la construcción social del lugar emergen aspectos poco conocidos y menos aun, analizados. Como ocurre con muchos otros hologramas, este muestra la construcción social de un tipo de lugares que suele estar presente en otras periferias metropolitanas 
excluidas de las grandes ciudades. Precisamente, su valor radica en que no es único, sino singular.

\section{Un holograma socioespacial sobre lugares casi invisibles}

Este holograma particular representa una ventana para la comprensión de lugares/espacios vividos parcialmente visibles o casi invisibles a pesar de su condición de outsideness (exterioridad) (Relph, 1976): se ancla en las calles de una periferia metropolitana excluida. La identificación de este holograma particular fue posible a partir de una preocupación teórica previa sobre paisajes y lugares invisibles, o lo que Joan Nogué (2005) denomina "las geografías de la invisibilidad".

La invisibilidad de un lugar que está fuera de un recinto -un outdoor (De Castro, 1997) - para quien allí habita o transita por el lugar parece indisociable de la siguiente pregunta: "Para quién es invisible lo que puede ser visible para otros" (Lindón, 2007). Una pregunta de este tipo no puede tener una respuesta única, sino muchas y específicas. Más allá de esas respuestas para cada contexto y situación, cabe destacar que la pregunta ubica el tema en una mirada: La invisibilidad, o la visibilidad parcial, de un outdoor no es independiente del punto de vista, no puede ser considerada al margen del sujeto que ve o no ve, ya que no se trata de una "invisibilidad estructural", sino de una invisibilidad o visibilidad experiencial. Por eso, más que lugares invisibles, son lugares parcialmente visibles (Nogué, 2005; 2006b; Lindón, 2007).

En nuestro trabajo sobre la periferia oriental excluida del Oriente de la ciudad de México, emergió un holograma en torno a un lugar particular: un basurero, era un área en la cual se alineaban una serie de grandes recipientes para la acumulación y posterior recolección de la basura. Sin embargo, para los jóvenes que habitaban el entorno, era el lugar de las experiencias sexuales. Indudablemente, para ellos también era el lugar donde se concentraba materialmente la basura, eso resultaba parte de lo evidente tanto para ellos como para cualquiera que por allí transitara o allí estuviera, por su materialidad insoslayable: la basura era una forma espacial, tanto la basura contenida en los recipientes como la basura dispersa en el suelo. Los perros, como los asiduos visitantes y exploradores del lugar, y los recipientes mismos de la basura completaban ese paisaje. Para el extranjero, el basurero era la forma espacial más visible, por ello no era difícil caracterizar al lugar como desolado y con carencias: Un lugar de la desolación y la basura. La idea de la desolación viene de la asociación con lo que falta en ese lugar, las carencias. Sin embargo, en términos estrictos esta visión de lo que falta procede de lo que allí está (la basura), que de inmediato nos permite ver al lugar como lo que no es, pero podría ser 6 . Por eso, para el forastero y también para muchos habitantes del lugar, parece un lugar del dolor y la aflicción por las carencias. Esta es la interpretación superficial (light), muy asociada a las formas espaciales en su expresión material.

Sin embargo, la consideración de que aquel lugar pueda ser interpretado como un holograma nos permitió iniciar el primer nivel de la interpretación espacial hologramática. Así, se hizo evidente que aquellas formas espaciales son inseparables de dimensiones no materiales, no visibles para otros, que no refieren ni a la desolación ni a las carencias sino a los encuentros sexuales de los jóvenes habitantes del lugar. En la experiencia espacial de aquel lugar, cada una de estas formas espaciales participa de maneras particulares para los jóvenes habitantes: Por ejemplo, los recipientes de basura constituyen una seudoprotección para una sexualidad efímera y nocturna. Los perros callejeros (perros homeless) son una compañía indiferente pero permanente de esa sexualidad. Así, la socialidad juvenil construye a ese espacio de la basura en un lugar de la sexualidad.

\footnotetext{
6 Nos referimos a ese rasgo propio del ser humano que Tuan identifica como el núcleo de una cultura y que se expresa en una forma de escapismo: El ser humano no solo ve el entorno, se somete, se adapta a él y lo transforma, sino también es capaz de "ver lo que no hay" (Tuan, 1998:24), indudablemente esto no es ajeno a la imaginación, a la capacidad de anticipar no solo cursos de acción, sino también un entorno espacial.
} 
Fue necesaria la narrativa de los propios jóvenes sobre ese lugar y sobre las experiencias allí vividas para que sus palabras lo hicieran visible. La clave fue la narrativa: la palabra que dio contenido al holograma, otorgó la visibilidad del lugar inicialmente no visible (Louiset, 2001) y superpuesto con otro visible, el de la desolación.

Al ser construido aquel arreglo espacial como un lugar de la sexualidad, por un lado esta se torna central para identificar y marcar el lugar: la sexualidad le da sentido al lugar y lo construye en un lugar de la sexualidad y la basura. Por otro lado, también ocurre lo inverso: la espacialidad es productora de la sexualidad; el espacio como producto de la sociedad y también productor de ella (Santos, 1990). En este caso el espacio sería el lugar de la basura y la sociedad, sería este tipo de sexualidad $Y$ como esa espacialidad en términos materiales está colmada de basura, la sexualidad toma rasgos de la basura, semántica y materialmente. Por ejemplo, la sexualidad es lo que queda (el residuo), es el residuo necesario que queda después de algo previo (deseo, impulso, una fiesta, un abandono, ejercicio del poder y la violencia...).

Igual que la basura, la sexualidad queda confinada espacialmente a la calle. Al mismo tiempo, esa sexualidad toma otros rasgos propios de ese espacio en sí, y no solo de la basura que en él se aloja. Así, la sexualidad es lo que se aloja en un espacio abierto en el cual cualquier objeto físico puede restar cierta visibilidad, pero también la sexualidad es lo que se vive ante los ojos de otros indiferentes. Estos rasgos propios de aquel espacio terminan siendo constitutivos de la sexualidad allí vivida. Además, el espacio también es constitutivo de la socialidad en otro sentido: se constata aquello que planteara Constancio de Castro (1997), en ciertos escenarios se pueden esperar algunos comportamientos reiterativos. En otras palabras, el lugar hace a la conducta. Para los jóvenes habitantes del lugar, desplazarse hacia este lugar en las noches implica ir en busca de una experiencia sexual.

En cuanto a la extensión temporal, los hologramas muestran que las prácticas de encuentros sexuales que sustentan esta construcción simbólica del lugar solo se desarrollan en condiciones nocturnas. Para quienes es visible este lugar tiene este sentido (está marcado) diurna y nocturnamente, aunque solo es practicado para la sexualidad nocturnamente.

En términos de la extensión espacial del lugar, el holograma muestra que el lugar se extiende en torno a los recipientes de basura y hasta ese mismo perímetro se extiende la construcción simbólica del lugar. Esto es relevante porque muestra que la construcción social y simbólica requiere necesariamente de las formas materiales dadas por los recipientes de basura. No obstante, eso no debería llevar a plantear un determinismo de las formas espaciales ya que es evidente la peculiar apropiación y refuncionalización de las formas: de contener la basura pasan a ser reapropiadas como un frágil muro que demarca "un recinto de sentido".

Con respecto al segundo plano de la interpretación espacial este holograma ofrece otro hallazgo relevante sobre la conexión con lugares distantes o al menos, diferentes al lugar narrado: el lugar de la sexualidad y la basura se conecta experiencialmente con lugares emplazados dentro de un recinto -indoor- o interioridades -insideness ${ }^{7}$ - de los hogares de estos jóvenes: en esas interioridades es usual es que la sexualidad de los adultos (padres) sea parte de las prácticas realizadas ante la mirada de los otros (los hijos, por ejemplo) y tenga un sentido semejante al de la sexualidad de la basura.

Este lugar de la basura y la sexualidad es invisible para los que no son parte de estas experiencias, por ello develar ese paisaje tiene un primer y evidente valor: como es el de desentrañar y reconocer lo aparentemen-

\footnotetext{
7 Para Relph, la interioridad o exterioridad no se anclan en construcciones materiales abiertas o cerradas, sino en el sentido del individuo por el lugar (Relph, 1976, p. 49-55). Lo interno y externo no derivan de estructuras materiales cerradas a modo de recinto o su ausencia, sino de la experiencia que el individuo tenga con ese lugar. Cuando los lugares carecen de sentido para la persona, habla de una "exterioridad existencial" que puede ser experimentada tanto en un lugar abierto como en uno cerrado, una casa por ejemplo.
} 
te no visible y no conocido. Sin embargo, más allá del hallazgo en sí, su importancia radica en que se constituye en una verdadera clave analítica para comprender otros aspectos de la vida social local a través de la relación con lugares diferentes y distantes: ciertos aspectos de las relaciones intradomésticas (las que ocurren en el indoor) se tornan más claros a partir de la forma en que aquel lugar fuera de un recinto (outdoor de la basura) configura la sexualidad. Esto no implica que la sexualidad del indoor sea resultado de la sexualidad que transcurre fuera del recinto (en los lugares casi invisibles), sino que estos últimos dan claves para comprender otros fenómenos asociados. Dicho con otras palabras, no se trata de considerar a ambos fenómenos en términos de causalidad sino que, al emerger uno de ellos (a través del holograma: los lugares de la sexualidad y la basura), permite comprender otros fenómenos y lugares asociados en alguna dimensión de la experiencia espacial (la sexualidad del indoor).

De este holograma se desprende una reflexión bifronte: por un lado, comprender lugares casi invisibles -o parcialmente visibles- requiere de acercamientos metodológicos ad hoc y "conceptos sensibilizadores" (Blumer, 1981), es decir, abiertos a captar la interpretación que hace la persona en cada situación. En este caso, el holograma mostró que aquello que aparentemente se podía concebir como un lugar de la desolación, el dolor y la basura, observado con profundidad devenía en un lugar de la sexualidad y la basura. Por ello, el desafío es desarrollar aproximaciones metodológicas -como los hologramas- que permitan hacer visibles los lugares casi invisibles, o lugares superpuestos debajo de otros muy visibles.

Por otro lado surge la otra cara de este mismo interrogante: como ciudadanos de la vida cotidiana -retomando la expresión de Habermas- cabe preguntarnos si nos interesa ver todos esos paisajes casi invisibles. O ¿será que la multiplicidad de la vida social, más aun de la vida metropolitana, nos hace optar por la invisibilidad de muchos lugares como una estrategia de vida? De aquí se desprende una hipótesis a explorar: esos lugares invisibles y superpuestos posiblemente estén construyendo un espacio de mucho espesor, en donde sobre un lugar se ha montado, invisiblemente para muchos, otro lugar, y sobre ese, otro y otro. Esto termina siendo una forma de vivir la multiplicidad: haciendo reducciones transitorias, es decir construyendo invisibilidades circunstanciales y situacionales. En otras palabras, ante una complejidad desbordante nos construimos la opción de no ver ciertos mundos, aun cuando estén junto a nosotros, como una forma de fragmentar selectivamente un mundo complejo.

\section{Reflexiones finales}

Actualmente, en Iberoamérica se ha ampliado el interés por comprender la espacialidad de la vida social, tanto a través de los aportes de la geografía humana, que se compromete con las vías constructivistas, ya sean las próximas al pensamiento francófono o al anglosajón (De Castro, 1997; Haesbaert, 1997; 2001; Hiernaux \& Lindon, 2006; Nogué, 1985; 1989; 1992; 1999; 2006a; 2007; Nogué \& Villanova, 1999; Santos, 1990; Uribe Castro, 2002; Yori, 1998; 1999), como por las visiones de las otras ciencias sociales, que también se preguntan por el espacio, el territorio y los lugares. Por ejemplo, se habla de una "sociología del lugar" (Gieryn, 2000). Todo este pensamiento social se halla en una circunstancia histórica particularmente favorable: el inicio de una perspectiva propia, pero al mismo tiempo, alimentada de todas las tradiciones mencionadas. Para este nuevo pensamiento iberoamericano sobre la espacialidad, la ventana de la construcción social del lugar se torna una alternativa de mucho interés así como los acercamientos cualitativos siempre asociados a esta. Precisamente, uno de los papeles más importantes de lo cualitativo para este constructivismo geográfico es la de canalizarlo hacia la investigación empírica y desde allí producir nuevas teorizaciones. Así, el constructivismo geográfico puede avanzar y al mismo tiempo, evitar el límite de la reflexión abstracta que se alimenta sobre sí misma.

Este constructivismo geográfico tiene la virtud de incorporar lo no material junto a lo material, así como comenzar a desprenderse del temor ancestral a que la centralidad del individuo pueda suponer la pérdida 
de lo social. Asimismo, también hay que subrayar que estas alternativas constructivistas parecen aligerar los lastres del pasado derivados de los fantasmas de los números, que tan fuertemente cerraron las posibilidades de comprender la subjetividad social y espacial. En síntesis, el constructivismo geográfico se atreve a explorar la espacialidad de la vida social a través del mundo del individuo ("Ios mundos interiores" de los que hablara Debarbieux, 1997) y a desprenderse de las ilusiones de verdad absoluta que habían prometido los números y las aproximaciones desde ellos cultivadas. Al hacerse cargo de esta osadía, el constructivismo geográfico encuentra en la investigación cualitativa una puerta de entrada a otra forma de producir conocimiento espacial, pero que al mismo tiempo permite estudiar aspectos que otras aproximaciones no podían focalizar.

Esto es relevante porque se renuevan las formas de concebir la realidad social y la espacialidad, pero también las formas de estudiarla (lo metodológico) e incluso emergen aspectos de la vida social y la espacialidad que para otras visiones no "existían" (Nogué, 1985). Un ejemplo pionero lo encontramos en el trabajo de Graham Rowles en donde mostró, que la espacialidad de las personas mayores lejos de reducirse a medida que aumenta la edad, en algunas ocasiones se amplía descomunalmente a través de lo que él denominó las "fantasías geográficas" (Rowles, 1978). Ese fenómeno que este autor denominó fantasía geográfica posiblemente existía desde tiempo atrás, sin embargo cuando los analistas solo "medían" las distancias físicas recorridas diariamente por estas personas no lo podían ver: era invisible.

En esta circunstancia histórica de apertura hacia el constructivismo geográfico, nuestra propuesta de los hologramas socioespaciales pretende plantearse solo como una forma de comenzar a abrir el camino. Esta propuesta no está libre de las dificultades propias de la interpretación, ni de ofrecer un conocimiento fragmentado. No obstante, estos dos rasgos no deben ser considerados como debilidades sino como particularidades de la propuesta. Lo relevante del conocimiento fragmentado que emerge de los hologramas es que en su esencia están las conexiones entre múltiples fragmentos. Por otra parte, lo fragmentado también es un intento por restituir el movimiento que es propio de la vida social, pero que las técnicas de investigación casi siempre han anulado. Como dijera Jesús Ibáñez (1994), la técnica es sedentaria pero la esencia de la técnica es nómada. Los hologramas socioespaciales aspiran a no perder de vista que la vida social que en ellos se despliega está en movimiento constante, es nómada.

Esta propuesta, al no llevar consigo los lastres del pasado, también tiene la virtud de abrirse a temas poco estudiados hasta ahora, como es el ejemplo de los lugares invisibles o parcialmente visibles, los espacios del miedo, los espacios de los homeless o del fenómeno conocido como homelessness, entre otros. En este contexto, la propuesta de los hologramas se plantea como una ventana hacia la reconstrucción de formas de espacialidad más complejas porque incluyen los experiencial. Así, para el constructivismo espacial los desafíos actuales son múltiples, y uno de ellos lo constituyen los lugares invisibles o parcialmente visibles. Los hologramas permiten, entre otras cosas, comprender cómo los sujetos llegan a construir un insideness (interioridad) dentro de un outsideness (exterioridad) (Relph, 1976), con la particularidad de que ese insideness solo es reconocido por quien participa de la situación o bien, quien tenga memoria espacial al respecto. El insideness es un "recinto de sentido" y no un recinto material, por eso no es visible para todos.

Estos recintos de sentido son creados a partir de la reapropiación de ciertos elementos materiales -de manera situacional-y de su resignificación, sin que ello traiga necesariamente modificaciones materiales. Las ciudades actuales encierran numerosos recintos de sentido parcialmente visibles, que a veces protegen a la persona mientras que otras condensan el dolor y la aflicción u otros sentidos del lugar muy diversos, pero que siempre se expresan en lo que hacen o evitan hacer los sujetos. Los hologramas socioespaciales asumen el desafío de hacer visibles estos lugares, y desentrañar el sentido de otros. Aunque esta propuesta la construimos a partir de la interpretación de una pe- 
riferia particular de la ciudad de México, ese tipo de lugares son frecuentes en otras ciudades. Pero más allá de la reproducción de los lugares de la basura, la propuesta del holograma se puede reconstruir con relación a otro tipo de lugares.

En nuestro ejemplo el holograma mostró como lo que era en apariencia un simple lugar de la basura, escondía otro lugar más complejo que articulaba dos mundos de no evidente articulación, como son la basura y la sexualidad. En casi todas las ciudades actuales hay interés por estudiar el problema de la basura, pero es visto a partir de las formas espaciales que esta genera. Sin embargo, el holograma presentado nos permite comprender prácticas y sentidos otorgados a los lugares de la basura no esperados desde miradas externas.

En suma, la propuesta de los hologramas busca constituir una alternativa -entre muchas otras- ante los grandes desafíos metodológicos que se han abierto a partir del momento en que el espacio y la espacialidad han comenzado a ser estudiados como lugares socialmente construidos, y no como localizaciones en un plano geométrico.

\section{Referencias bibliográficas}

BAILLY, A. Lo imaginario espacial y la geografía: En defensa de la geografía de las representaciones. Anales de Geografía de la Universidad Complutense, 1989, № 9, p. 11-19, 1989.

BARNES, T. \& GREGORY, D. Textuality and human geography. In: TREVOR, B. \& GREGORY, D. (eds.) Reading in human geography: The Poetics and politics of inquiry, London: Arnold, 1997, p. 138-144.

BLUMER, H. El interaccionismo simbólico: Perspectiva y método. Barcelona: Hora, 1981.

BONNEMAISON, J. La géographie culturelle. Paris: Editions du C.T.H.S, Ministère de I'Education Nationale, 2000.

BUTTIMER, A. Grasping the dynamism of lifeworld. Annals of the American Geographers, 1976, № 2, Vol. 66, p. 277-292.
BUTTIMER, A. \& SEAMON, D. The human experience of space and place. New York: St. Martin Press, 1980.

BUTZ, D. \& EYLES, J. Reconceptualizing senses of place: Social relations, ideology and ecology. Geografiska Annaler, 1997, № 79, Serie B, p. 1-25.

CHEVALIER, J. Espace de vie ou espace vécu? L'Espace Géographique, 1974, № 1, p. 1-3.

COLLIGNON, B. \& STASZAK, J-F. (eds.). Espaces domestiques: Construire, habiter, représenter. Paris: BREAL, 2003.

COSGROVE, D. Prospect, perspective and the evolution of the landscape idea. Transactions of the Institute of British Geographers, 1985, № 1, Vol. 10, p. 45-62.

COSGROVE, D. Observando la naturaleza: El paisaje y el sentido europeo de la vista. Boletín de la A.G.E, 2002, № 34, p. 63-89.

DANIELS, S. Arguments for a humanistic geography. In: BARNES, T. \& GREGORY, D. (eds.) Reading in human geography: The poetics and politics of inquiry. London: Arnold, 1997, p. 364-375.

DARDEL, E. L'homme et la terre: Nature de la réalité géographique. Paris: Editions du CTHS, 1990.

DE CASTRO, C. La geografía en la vida cotidiana. Barcelona: Ediciones del Serbal, 1997.

DEBARBIEUX, B. Le lieu, fragment et symbole du territoire. Espaces et Sociétés, 1995, № 82-83, p. 13-36.

DEBARBIEUX, B. L'exploration des mondes intérieurs. In: KNAFOU, R. (ed.) L'état de la géographie. Paris: Belin, 1997, p. 371-384.

DI MEO, G. L'Homme, la société, I'espace. Paris: Anthropos, 1991.

DI MEO, G. Géographies tranquilles du quotidien: Une analyse de la contribution 
des sciences sociales et de la géographie à l'étude des pratiques spatiales. Cahiers de Géographie du Québec, 1999, № 118, Vol. 43, p. 75-93.

DI MEO, G. Que voulons-nous dire quand nous parlons d'espace?. In: LÉVY, J. \& LUSSAULT, M. (eds.) Logiques de I'espace: Esprit des lieux. Géographies à Cerisy. Paris: Belin, 2000a, p. 37-48.

DI MEO, G. Géographie sociale et territoires. Paris: Nathan, 2000b.

DUNCAN, J. \& LEY, D. Introduction: Representing the place of culture. In: DUNCAN, J. \& LEY, D. (eds.) Place/Culture/Representation. London: Routledge, 1993, p. 1-24.

ENTRIKIN, J. The betweenness of place: Towards a geography of modernity. Baltimore: Johns Hopkins University Press, 1991.

ENTRIKIN, J. Contemporary humanism in geography. Annals of the Association of American Geographers, 1976, № 66, p. 615-632.

EYLES, J. Senses of place. Warrington: Silverbrook Press, 1985.

EYLES, J. Interpreting the geographical world. In: EYLES, J. \& SMITH, D. (eds.) Qualitative methods in human geography. Oxford: Polity Press-Basil Blackwell, 1988, p. 1-16.

FRÉMONT, A. La région: Espace vécu. Paris: Flammarion, 1999.

FRÉMONT, A. Aimez-vous la géographie? Paris: Flammarion, 2005.

GIERYN, T. A space for place in sociology. Annual Review of Sociology, 2000, № 1 , Vol. 26, p. 463-496.

GREGORY, D. Lefebvre, Lacan and the production of space. In: BENKO, G. \& STROHMAYER, U. (eds.) Geography, history and social sciences. Dordrecht: Kluwer Academic Publishers, 1995, p. 15-44.

GUMUCHIAN, H. Représentations et aménagement du territoire. París: Economica, 1991.
GUMUCHIAN, H.; GRASSET, E.; LAJARGE, R. \& ROUX, E. Les acteurs, ces oubliés du territoire. Paris: Anthropos-Economica, 2003.

HAESBAERT, R. Des-territorialização e identidade: A rede "gaúcha" no nordeste. Niterói, Rio Janeiro: Editora da Universidade Federal Fluminense, 1997.

HAESBAERT, R. Território, cultura e desterritorialização. In: ROSENDAHL, Z. \& LOBATO CORRÊA, R. (eds.) Religião, identidade e territorio, Rio de Janeiro: EdUERJ, 2001, p. 50-82.

HARLEY, B. La nueva naturaleza de los mapas. México: FCE, 2005.

HIERNAUX, D. Metrópoli y etnicidad. Los indígenas en el Valle de Chalco. México: El Colegio Mexiquense-FONCA, 2000.

HIERNAUX, D. Paisajes fugaces y geografías efímeras en la metrópolis contemporánea. In: NOGUÉ, J. (ed.) La construcción social del paisaje. Madrid: Editorial Biblioteca Nueva, 2007, p. 237-258.

HIERNAUX, D. \& LINDÓN, A. Repensar la periferia: De la voz a las visiones exo y egocéntricas. In: AGUILAR, A. (eds.) Procesos metropolitanos y grandes ciudades: Dinámicas recientes en México y otros países. México: Instituto de Geografía-Miguel Angel Porrúa, 2004, p. 413-443.

HIERNAUX, D. \& LINDÓN, A. (eds.) Tratado de Geografía Humana. Barcelona: Anthropos-UAM-I, 2006.

IBAÑEZ, J. El regreso del sujeto: La investigación social de segundo orden. Madrid: Siglo XXI, 1994.

JAVEAU, C. Lugares de memoria individuales y estructuración de las interacciones: Acerca de los síndromes de Lamartine y de Proust. In: LINDÓN, A. (ed.) La vida cotidiana y su espacio-temporalidad. Barcelona: Anthropos-CRIM-El Colegio Mexiquense, 2000, p. 171-186.

LEY, D. Social geography and social action. In: LEY, D. \& SAMUELS, M. (eds.) 
Humanistic geography: Prospects and problems. London: Croom-Helm, 1978, p. 41-57.

LEY, D. Behavioural geography and the philosophies of meaning. In: COX, K. \& GOLLEDGE, R. (eds.) Behavioural problems in geography revisited. London: Methuen, 1981a, p. 209-230.

LEY, D. Cultural/Humanistic Geography. Progress in Human Geography, 1981b, № 5, p. 249-257.

LEY, D. Interpretative social research in the inner city. In: EYLES, J. (ed.) Research in Human Geography. Oxford: Basil Blackwell, 1988, p. 121-138.

LEY, D. Fragmentation, coherent and the limits to theory in human geography. In: KOBAYASHI, A. \& MACKENZIE, S. (ed.) Remarking human geography. London: Unwin Hyman, 1989, p. 227-244.

LINDÓN, A. Utopías, atopías y construcción del lugar: La periferia oriental de la ciudad de México. Ciudades, 2003, № 60, p. 48-54.

LINDÓN, A. Figuras de la territorialidad en la periferia metropolitana: Topofilias y topofobias. In: REGUILLO, R \& GODOY, M. (eds.) Ciudades translocales: Espacios, flujo y representación. Perspectivas desde las Américas. Guadalajara: SSRC-ITESO, 2005a, p. 145-172.

LINDÓN, A. El imaginario suburbano: topofilias y topofobias. Cidades, 2005b, № 5, Vol. 2, p. 289-314.

LINDÓN, A. Geografías de la vida cotidiana. In: HIERNAUX, D. \& LINDÓN, A. (eds.) Tratado de Geografía Humana. BarceIona: Anthropos-UAM-I, 2006a, p. 477-536.

LINDÓN, A. La casa bunker y la deconstrucción de la ciudad. Liminar. Estudios sociales y humanísticos, 2006b, № 2, Año 4, Vol. IV, p. 18-35.

LINDÓN, A. La construcción social de paisajes invisibles y del miedo. In: NOGUÉ, J. (ed.) La construcción social del paisaje.
Madrid: Editorial Biblioteca Nueva, 2007, p. 213-236.

LINDÓN, A. \& HIERNAUX, D. La Geografía Humana: Un camino a recorrer. In: HIERNAUX, D. \& LINDÓN, A. (eds.) Tratado de Geografía Humana. Barcelona: Anthropos-UAM-I, 2006, p. 1-22.

LOUISET, O. Les villes invisibles. L'Information Géographique, 2001, № 653, p. 135-152.

MARCONIS, R. Introduction à la géographie. Paris: Armand Colin, 1996.

MINCA, C. Más allá del posmodernismo: Viaje a través de la paradoja moderna. Documents d'Analisi Geografica, 2002, № 40, p. 45-68.

MONDADA, L. Décrire la ville: La construction des savoirs urbains dans l'interaction et dans le texte. Paris: Anthropos, 2000.

NOGUÉ, J. Une méthode de travail humaniste $=\mathrm{A}$ humanistic work-method. Documents d'Anàlisi Geogràfica, 1985, № 6, p. 67-80.

NOGUÉ, J. Espacio, lugar, región: Hacia una nueva perspectiva geográfica regional. Boletín de la AGE, 1989, № 9, p. 49-62.

NOGUÉ, J. El paisaje existencial de cinco grupos de experiencia ambiental: Ensayo metodológico. In: GARCÍA BALLESTEROS, A. (ed.) Geografía y humanismo. Barcelona: Oikos-Tau, 1992, p. 87-96.

NOGUÉ, J. El retorno al lugar: La creación de identidades territoriales. Claves de la razón práctica, 1999, № 92, p. 9-11.

NOGUÉ, J. Las geografías de la invisibilidad. In: Paisatges incògnits, territoris ocults: les geografies de la invisibilitat. Iller Seminari internacional sobre paisatge: Paisajes incógnitos, territorios ocultos: Las geografías de la invisibilidad. Olot: Observatori del Paisatge-CUIMPB, 2005. Disponible en Internet: http://www.catpaisatge.net/fitxers/ 2005_joan_nogue.pdf

NOGUÉ, J. Paisaje, identidad nacional y sociedad civil en la Cataluña contemporá- 
nea. In: LÓPEZ, A.; NOGUÉ, J. \& ORTEGA, N. (eds.) Representaciones culturales del paisaje: $Y$ una excursión por Doñana. Madrid: UAM-AGE, 2006a, p. 41-58.

NOGUÉ, J. (ed.). La construcción social del paisaje. Madrid: Editorial Biblioteca Nueva, 2007.

NOGUÉ, J. \& VILLANOVA, J. (eds.) España en Marruecos (1912-1956): Discursos geográficos e intervención territorial. Lleida: Editorial Milenio, 1999.

NORA, P. Les lieux de mémoire. Tomo I. Paris: Gallimard, 1997.

OLSSON, G. Of ambiguity or far cries from a memorializing. In: LEY, D. \& SAMUELS, M. (eds.) Humanistic geography: Prospects and problems. London: CroomHelm, 1978, p. 109-120.

OLSSON, G. Birds in egg/eggs in bird. London: Pion, 1980.

OLSSON, G. Lines of power/Limits of language. Minneapolis: University of Minnesota Press, 1991.

OLSSON, G. Misión imposible. Anales de Geografía de la Universidad Complutense, 1997, № 17, p. 39-51.

PHILO, C. Más palabras, más mundos: Reflexiones en torno al 'giro cultural' y a la geografía social. Documents d'Analisi Geografica, 1999, № 34, p. 81-99.

PRATT, G. Spatial metaphors and speaking positions. In: BARNES, T. \& GREGORY, D. (eds.) Reading in human geography: The poetics and politics of inquiry. London-Nueva York: Arnold, 1997, p. 168-172.

PRED, A. Social reproduction and the time-geography of everyday life. Geografiska Annaler, Serie B, Human Geography, 1981, № 1, Vol. 63, p. 5-22, 1981.

PRED, A. Place as historically contingent process: Structuration and the time-geography of becoming places. Annals of the Association of American Geographers, 1984, № 2, Vol. 74, p. 279-297.
REAL ACADEMIA ESPAÑOLA (RAE). Diccionario de la lengua española. Vigésima Primera. Edición Madrid: Espasa, 1992.

RAFFESTIN, C. Paysage et territorialité. Cahiers de Géographie de Québec, 1977, № 21, p. 123-134.

RAFFESTIN, C. Remarques sur les notions d'espace, de territoire et de territorialité. Espace et Société, 1982, № 41, p. 167-171.

RAFFESTIN, C. Ecogenèse territoriale et territorialité. In: AURIAC, F. \& BRUNET, R. (eds.) Espace, jeux et enjeux. Paris: FayardFondation Diderot, 1986, p. 172-185.

RELPH, E. Place and placelessness. London: Pion, 1976.

ROWLES, G. Reflections on experiential fieldwork. In: LEY, D. \& SAMUELS, M. (eds.) Humanistic geography: Prospects and problems. London: Croom-Helm, 1978, p. 173-193.

SACK, R. Conceptions of space in social thought: A geography perspective. Minneapolis: University of Minnesota Press, 1980.

SACK, R. Human territoriality: Its theory and history. Cambridge: Cambridge University Press, 1986.

SANTOS, M. Por una geografía nueva. Madrid: Espasa Universidad, 1990.

SOJA, E. Thirdspace: Journey to Los Angeles and other real-and-imagined places. Mass: Ed. Blackwell, 1996.

TUAN, Y. Space and place: The perspective of experience. London: Edward Arnold, 1977.

TUAN, Y. Landscapes of fear. New York: Pantheon-Blackwell's, 1980.

TUAN, Y. Topophilia: A study of environmental perception, attitudes, and values. New York: Columbia University Press, 1990.

TUAN, Y. Escapismo: Formas de evasión en el mundo actual. Barcelona: Península, 1998. 
TURCO, Á. Pragmatiques de la territorialité: Compétence, science, philosophie. In: LÉVY, J. \& LUSSAULT, M. (eds.) Logiques de l'espace: Esprit des lieux. Géographies à Cerisy. Paris: Belin, 2000, p. 287-299.

URIBE CASTRO, H. El lugar: entre candados, rejas y miedos. Biblio $3 W$, Revista Bibliográfica de Geografía y Ciencias Sociales, 2002, № 393, Vol. VII.

YORI, C. La Topofilia como una estrategia para hacer ciudad desde sus habitantes.
Cuadernos de Estudios Urbanos, Construcción socio-cultural del espacio urbano. Bogotá: Editorial Corporación de Estudios Urbanos, 1998, № 2.

YORI, C. Topofilia o la dimensión poética del habitar. Bogotá: Centro Editorial Javeriano CEJA e Instituto Colombiano para el Fomento a la Ciencia y la Tecnología, 1999.

ZENEIDE-HENRY, D. Les SDF et la ville: Géographie du savoir vivre. Paris: BREAL, 2002. 
1 